\title{
PERFIL FUNCIONAL DA PARALISIA FACIAL EM UM HOSPITAL
}

\author{
Katia Mayumi Konno ${ }^{a}$ \\ Marise Bueno Zonta ${ }^{b}$ \\ Hélio A G. Teive ${ }^{c}$ \\ Clynton Lourenço Correa ${ }^{d}$
}

\begin{abstract}
Resumo
A paralisia do nervo facial pode ser central ou periférica, sendo a paralisia nervosa mais acometida do corpo humano. O objetivo deste estudo foi estabelecer o perfil funcional dos pacientes com paralisia facial internados no Setor de Neurologia do Hospital das Clínicas da UFPR. Por meio de estudo prospectivo, com amostragem por conveniência e aplicação de um questionário para coleta dos dados clínicos, foi realizada a identificação do paciente e avaliação do grau de comprometimento muscular que foi classificada de acordo com a escala de evolução motora de House-Brackmann pelo período de 12 meses. Participaram do estudo 50 pessoas que representaram $9 \%$ dos internados na unidade. A etiologia predominante da paralisia facial foi vascular, com $88 \%$ dos casos de origem central. Foi observado padrão sazonal para a paralisia facial central com maior prevalência nos meses de abril à julho e paralisia facial periférica entre os meses de maio à julho. Diferentemente de outros estudos, não foi relatada queixa em relação à aparência. Apesar de existir encaminhamento à fisioterapia foi observada a necessidade de estabelecer um fluxograma para reabilitação dos pacientes durante o internamento e no pós-alta.
\end{abstract}

Palavras-chave: Fisioterapia; Paralisia Facial; Reabilitação.

Correspondence Author: Katia Mayumi Konno - katiakonno@gmail.com

a. Fisioterapeuta e Residente do Programa de Residência Multiprofissional do Hospital das Clínicas - Universidade Federal do Paraná.

b. Fisioterapeuta Doutora do Hospital das Clínicas - Universidade Federal do Paraná.

c. Neurologista, Professor Doutor Adjunto do curso de Medicina da Universidade Federal do Paraná.

d. Fisioterapeuta, Professor Doutor Adjunto do curso de Fisioterapia da Universidade Federal do Rio de Janeiro. 


\title{
FUNCTIONAL PROFILE OF THE FACIAL PALSY FROM A HOSPITAL
}

\begin{abstract}
The facial nerve paralysis may be central or peripheral, being a major nerve paralysis of the human body. The aim of this study was to establish the functional profile of patients with facial paralysis admitted to the Neurology Department of the Hospital at the University Federal of Paraná. In a prospective study with convenience sampling and a questionnaire to collection of clinical data, patient identification and assessment of the degree of muscle impairment. This work was performed at a period by 12 months and the data was classified according to the scale of motor progress of House-Brackmann. The study included 50 people who represented $9 \%$ of inpatients in the unit. The predominant etiology of facial paralysis was vascular in $88 \%$ of cases of central origin. Seasonal pattern was observed for the central peripheral paralysis most prevalent from April to July and peripheral facial paralysis between the months of May to July. Unlike other studies, were not reported complaints over appearance. Although there is referral to physiotherapy the need to establish a flowchart for rehabilitation of patients during hospitalization and post-discharge was observed.
\end{abstract}

Keywords: Physical Therapy Specialty; Facial Palsy; Rehabilitation.

\section{INTRODUÇÃO}

O nervo facial ou VII par craniano, responsável pelas expressões da face, lacrimação, salivação e percepção de sensações gustativas, é, entre todos os nervos do corpo, o que apresenta paralisia com maior freqüência. (1) Suas desordens são causadas por diferentes doenças incluindo as circulatórias, traumáticas, infecciosas e tumorais. Dependendo do local do trauma a lesão do nervo facial pode resultar em uma aparência distorcida da face durante o repouso ou execução de movimento voluntário, (2) comprometendo a expressão facial, fundamental para a comunicação. ${ }^{(3,4)}$ A Paralisia Facial (PF) é comumente classificada como "neurônio motor superior", no caso da origem central, ou como "neurônio motor inferior", quando periférica. (5)

A lesão mais frequente do neurônio motor superior envolvendo o VII par craniano é decorrente do acidente vascular encefálico (AVE), que compromete os neurônios do córtex motor ou, de forma mais frequente, seus axônios na cápsula interna. $(2,5,6,7)$
A etiologia das lesões do neurônio motor inferior é desconhecida em aproximadamente metade dos casos, sendo a de Bell (ou idiopática), a de maior incidência. ${ }^{(8)}$

A recuperação dos movimentos na PFP e na PFC dependerá do nível da lesão do nervo facial e do trato córtico-bulbar, respectivamente, podendo ser parcial ou total. ${ }^{(2)}$ Outros fatores como etiologia, idade do paciente, comprometimento neuromuscular, tipo de lesão do nervo e o tratamento instituído podem contribuir para a recuperação do nervo facial. ${ }^{(9,10,11)}$ Aproximadamente $20 \%$ dos casos evoluem com alguma sequela, que pode variar do grau leve até a paralisia completa dos movimentos dos músculos faciais. ${ }^{(5)}$

A intervenção de uma equipe multidisciplinar de reabilitação é indispensável para a recuperação dos movimentos faciais e suas funções. ${ }^{\left({ }^{(2)}\right)}$ Os recursos sugeridos pela literatura incluem a cinesioterapia, massagem e eletrotermoterapia. ${ }^{(10,11)}$ 
A abordagem fisioterapêutica tem como objetivo restabelecer o trofismo, a força e a função muscular, podendo adequar a qualidade de vida do paciente a uma sequela neurológica. ${ }^{(2)}$

Diferentes aspectos da PF têm sido abordados na literatura científica, mas não a caracterização da frequência e apresentação clínica em unidade de internação. O objetivo deste estudo foi estabelecer o perfil funcional dos pacientes com PFC e PFP atendidos na Unidade de Internamento do Setor de Neurologia do Hospital das Clínicas da UFPR.

\section{MÉTODO}

Este trabalho foi aprovado pelo Comitê de Ética em Pesquisa (CEP) do Hospital das Clínicas da Universidade Federal do Paraná (HC-UFPR), segundo as determinações do Conselho Nacional de Saúde (Resolução ${ }^{\circ} 466$, de 12 de dezembro de 2012), recebendo sua aprovação sob o registro CEP 2542.149/2011-06. Os participantes foram esclarecidos sobre o objetivo deste estudo e convidados a participar do mesmo, o qual foi iniciado após a concordância e a assinatura do Termo de Consentimento Livre e Esclarecido.

O estudo foi prospectivo com amostragem por conveniência na Unidade de Internamento da Neurologia - HC. Foram convidados a participar do estudo todos os pacientes com idade superior a 18 anos, admitidos na Unidade de Internamento da Neurologia no período de 12 meses (09/2011 a 09/2012) que apresentassem PFC ou PFP. Foram excluídos os pacientes com déficit do nível de consciência ou que não concordaram em participar da pesquisa.

A coleta de dados foi realizada através de um protocolo contendo dados clínicos e de identificação colhidos do prontuário médico, e dados da avaliação fisioterapêutica. Os dados clínicos observados foram: etiologia, diagnóstico clínico e doenças prévias.

A avaliação fisioterapêutica considerou as queixas funcionais como lacrimejamento e perda de alimento, e a avaliação clínica dos sinais e sintomas da paralisia. Os sinais e sintomas observados foram presença de hiperacusia, simetria facial em repouso e em movimento, observando o nivelamento dos olhos, do septo nasal e comissura labial e sincinesias. A qualidade do tônus muscular foi avaliada pela palpação manual realizando-se movimentos passivos em toda a face do paciente e graduada pela escala de evolução motora de House-Brackmann (HB). ${ }^{(13)}$

A análise estatística dos dados foi descritiva onde as variáveis qualitativas foram apresentadas em frequências relativas (percentuais) e frequências absolutas (n), e as variáveis quantitativa- numérico em média e desvio padrão.

\section{RESULTADOS}

Durante o período considerado no estudo internaram na Unidade de Neurologia 569 pacientes para investigação neurológica. Destes, 50 pacientes, representando $9 \%$ dos internados, foram incluídos no estudo por apresentar a PF, sendo 44 (88\%) PFC e 6 (12\%) PFP. Dos avaliados 26 (52\%) eram do gênero masculino. A média de idade foi de 58 anos $( \pm 15,7)$, variando entre 20 a 95 , com mediana de 60 anos.

Nesta amostra, dos 44 (88\%) indivíduos que apresentaram a PFC, 29 (66\%) eram de etiologia vascular ocasionados por Acidente Vascular Encefálico isquêmico (AVEi), 9 (20\%) por Acidente Vascular Encefálico hemorrágico (AVEh) e 1 (2\%) por Acidente Isquêmico Transitório. A PFP, observada em $6(12 \%)$ casos, eram de etiologia desconhecida e em apenas 1 caso foi observado diagnóstico de doença de Guillain-Barré.

Observou-se que 27 (62\%) dos casos de PFC ocorreram nos meses de abril a julho, o que caracteriza uma apresentação sazonal (Figura 1). Característica semelhante foi observada na PFP onde $5(84 \%)$ também ocorreram nos meses de maio a julho. 


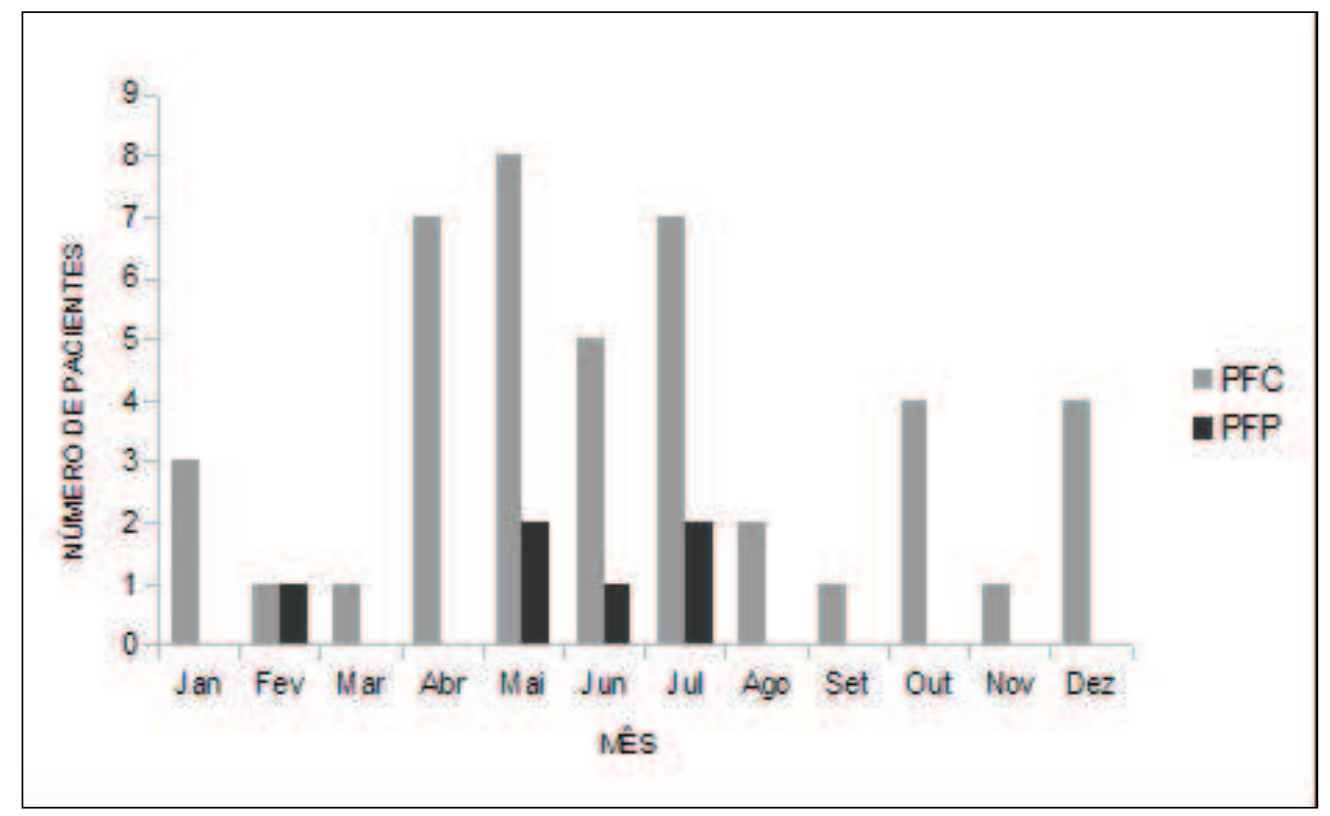

Figura 1 - Distribuição temporal da Paralisia Facial Central $(n=44)$ e Periférica $(n=6)$ do setor de internamento da Neurologia no período de doze meses.

No momento do internamento todos os casos de PF estavam associados à outras comorbidades. Foram observadas comorbidades prévias clinicamente diagnosticadas em 41 (82\%) dos casos, sendo que $33(66 \%)$ indivíduos apresentavam Hiper- tensão Arterial Sistêmica (HAS) e 8 (16\%) outras doenças de origem nervosa como esclerose múltipla, doença de Parkinson e neoplasias. A prevalência das queixas e sinais característicos da PF nesta amostra estão descritos na Tabela 1.

Tabela 1 - Sinais e Sintomas Característicos da Paralisia Facial Central e Periférica do setor de internamento da Neurologia no período de doze meses

\begin{tabular}{ccc}
\hline PFC & PFP & SINAIS E SINTOMAS \\
\hline$(\mathrm{N}=44)$ & $(\mathrm{N}=6)$ & Assimetria \\
$86 \%$ & $100 \%$ & Sialorréia \\
$82 \%$ & $67 \%$ & Sincinesia \\
$16 \%$ & $67 \%$ & Lacrimejamento \\
$11 \%$ & $67 \%$ & Hiperacusia \\
$4 \%$ & $17 \%$ & Principais Queixas \\
- & - & Perda de alimento \\
$68 \%$ & $66 \%$ & Dificuldade para mastigar \\
$57 \%$ & $50 \%$ & Lesões internas da boca \\
$18 \%$ & $33 \%$ & Incômodo ocular \\
$11 \%$ & $50 \%$ &
\end{tabular}

Durante a permanência na unidade de internamento da neurologia, 44 (80\%) pacientes foram encaminhados ao serviço de Fisioterapia. Deste total,
38 (86\%) foram encaminhados para reabilitação externa após alta hospitalar. As PF foram recorrentes em 6 (12\%) dos casos, sendo 3 de origem vascular 
por recorrência do AVE e 3 de origem periférica sem causa definida.

Em relação ao quadrante afetado, 8 (16\%) apresentaram envolvimento de toda hemiface direita, com ambos os quadrantes, superior e inferior afetados, e em 17 (34\%) apenas o quadrante inferior direito. Toda a hemiface esquerda estava acometida em 5 (10\%) pacientes, e em 19 (38\%) dos casos apenas o quadrante inferior esquerdo.

O comprometimento dos músculos nas PFC, avaliado pela escala de $\mathrm{HB}$, foi predominante no quadrante inferior da face (Tabela 2). O músculo risório foi o que apresentou maior comprometimento (grau $\mathrm{V} \mathrm{e} \mathrm{VI)} \mathrm{em} 44 \%$ dos casos, seguidos pelo levantador do lábio superior, zigomático maior e menor, depressor do lábio inferior e os elevadores do ângulo da boca (40\%), orbicular da boca (39\%) e levantador do lábio superior e da asa do nariz (38\%). Os comprometimentos leves foram predominantes nos quadrantes superiores da face.

Nas PFP o comprometimento muscular moderado a grave foi predominante nos músculos levantador do angulo da boca e zigomático maior e menor, levantador do lábio superior e da asa do nariz, levantador do lábio superior e depressor do lábio inferior. Os dados referentes à avaliação do grau de comprometimento do nervo facial nos casos PFC e PFP pela escala de evolução motora de HB estão apresentados na Tabela 2.

Tabela 2 - Avaliação do comprometimento do nervo facial nas Paralisias Faciais Centrais e Periféricas segundo a escala de evolução motora de House-Brackmann

\begin{tabular}{|c|c|c|c|c|c|c|}
\hline & $\begin{array}{c} \\
\text { PFC/PFP } \\
\end{array}$ & $\begin{array}{c}\mathrm{II} \\
\text { PFC/PFP } \\
\end{array}$ & $\begin{array}{c}\text { III } \\
\text { PFC/PFP }\end{array}$ & $\begin{array}{c}\mathrm{IV} \\
\mathrm{PFC} / \mathrm{PFP}\end{array}$ & $\begin{array}{c}\mathrm{V} \\
\text { PFC/PFP }\end{array}$ & $\begin{array}{c}\mathrm{VI} \\
\mathrm{PFC} / \mathrm{PFP}\end{array}$ \\
\hline Occipitofrontal & $57 / 50$ & $14 / 17$ & $7 / 0$ & $2 / 33$ & $2 / 0$ & $7 / 0$ \\
\hline Corrugador do supercílio & $50 / 50$ & $20 / 17$ & $11 / 0$ & $\mathrm{O} / 33$ & $5 / 0$ & $2 / 0$ \\
\hline Orbicular do olho & $36 / 33$ & $30 / 50$ & $11 / 17$ & $5 / 0$ & $2 / 0$ & $5 / 0$ \\
\hline $\begin{array}{l}\text { Levantador do ângulo boca, } \\
\text { Zigomático Maior e Menor }\end{array}$ & $2 / 17$ & $20 / 17$ & $9 / 17$ & $18 / 17$ & $20 / 33$ & $20 / 0$ \\
\hline Levantador do lábio superior e da asa do nariz & $7 / 17$ & $18 / 17$ & $11 / 33$ & $16 / 17$ & $18 / 33$ & $20 / 0$ \\
\hline Levantador lábio superior & $5 / 17$ & $25 / 0$ & $7 / 33$ & $11 / 17$ & $20 / 33$ & $23 / 0$ \\
\hline Depressor do lábio inferior & $7 / 33$ & $25 / 0$ & $7 / 17$ & $14 / 0$ & $20 / 50$ & $20 / 0$ \\
\hline Orbicular da boca & $2 / 17$ & $25 / 0$ & $11 / 33$ & $9 / 0$ & $16 / 33$ & $23 / 17$ \\
\hline Risório & $7 / 17$ & $18 / 0$ & $14 / 33$ & $14 / 17$ & $14 / 17$ & $30 / 17$ \\
\hline Mentoniano & 717 & $23 / 0$ & $7 / 33$ & $18 / 33$ & $14 / 33$ & $23 / 17$ \\
\hline Prócero & $43 / 17$ & $23 / 17$ & $11 / 17$ & $2 / 17$ & $5 / 17$ & $5 / 17$ \\
\hline Platisma & $18 / 17$ & $20 / 33$ & $9 / 17$ & $18 / 17$ & $14 / 17$ & $11 / 0$ \\
\hline Depressor do ângulo da boca & $5 / 17$ & $25 / 33$ & $7 / 0$ & $18 / 0$ & $16 / 17$ & $20 / 0$ \\
\hline Bucinador & $2 / 17$ & $25 / 33$ & $16 / 0$ & $11 / 17$ & $23 / 33$ & $14 / 17$ \\
\hline
\end{tabular}

\section{DISCUSSÃO}

O presente estudo foi realizado na unidade de internação de um hospital universitário e de alta complexidade, o que pode justificar a maior prevalência da PFC neste trabalho devido ao internamento de pacientes acometidos por AVE. A PFP apresentou menor incidência, pois eram pacientes eletivos, sem diagnóstico específico e estavam em investigação clínica. 
Encontrou-se um leve predomínio de indivíduos do sexo masculino, com $52 \%$ dos casos acometidos. Em outros trabalhos, foram observados predomínios semelhantes, porém não foram observadas diferenças significativas em relação ao gênero. ${ }^{(6,14)}$

A etiologia vascular foi predominante neste trabalho principalmente devido ao AVEi. A PFC tem por principal etiologia as doenças cerebrovasculares, ${ }^{(2,5,6,7)}$ e o aumento da incidência da PFC a partir dos 60 anos de idade, pode ser devido a esta faixa etária ser a mais acometida por doenças crônicas não transmissíveis, com destaque para as doenças cardiovasculares ${ }^{15}$. Atualmente, as doenças cardiovasculares são as principais causas de óbitos no Brasil e o maior predisponente do AVE. ${ }^{(2)}$

A presença da PFC, de etiologia vascular, foi observada em $57 \%$ dos casos com idade acima dos 60 anos, e em $36 \%$ compreendendo as faixas etárias entre 40 a 59 anos, condizente com a maior prevalência dos AVE nestas faixas etárias. Diferentes estudos verificaram que entre as PFP não foram observadas diferenças significativas em relação à idade. ${ }^{(16,17,18)}$ Neste estudo o desvio da idade pela PFP foi alto, o que evidenciou ausência de um padrão de faixa etária para a PFP, o que condiz com os resultados da literatura acima citada.

Vários estudos epidemiológicos abordam o aspecto da sazonalidade na ocorrência da PF e concluíram uma maior predisposição nos períodos frios. ${ }^{(19)}$ Esta característica sazonal foi observada na ocorrência da PFC e da PFP abordada por este estudo, onde as maiores incidências foram nos meses de maio a julho (Figura 1). A etiologia da PFC foi predominantemente vascular nos casos observados, principalmente devido ao AVEi. Estudos verificaram a existência da sazonalidade nas ocorrências dos AVE, principalmente o AVEi que é predominante nas estações de outono e inverno. Em outros estudos, foi observada associação entre sazonalidade dos AVEi e a idade, onde indivíduos com idade inferior a 60 anos demonstraram maior sensibilidade a variação sazonal, sendo significativamente mais propensos a desenvolver AVE nos períodos de inverno e primavera. ${ }^{(21,22)}$ Neste estudo
$37 \%$ dos casos de PF contemplavam idades entre 40 a 59 anos que compreende a faixa etária, teoricamente sensível à variação sazonal, observada nestes trabalhos. As razões biológicas para a maior incidência de AVEi durante os períodos frios não são conhecidas, porém um estudo(23) refere que dentre os fatores envolvidos a vasoconstrição periférica (causada pelo frio) e consequente aumento da pressão sanguínea possa estar relacionado a causalidade do AVE nesses períodos. Além disso, os níveis de colesterol total e triglicéridos também tendem a ser elevados neste período. Apesar da maioria dos estudos indicar prevalência nos períodos frios, pesquisadores ${ }^{(21)}$ sugerem que a predisposição possa estar relacionada a exposição a temperaturas extremas, seja fria ou quente.

As PFP contemplaram, em sua maioria, os meses de maio a julho, o que corresponde ao período de outono e inverno do hemisfério sul (Figura 1). Alguns autores encontraram menor prevalência no verão para paralisia periférica de Bell ${ }^{(19,20)}$ e maior incidência no final do inverno e no início da primavera ${ }^{21}$. Estudos propõem que temperaturas frias possam predispor a PFP idiopática, devido às infecções virais frequentes deste período. ${ }^{(20)}$ Contudo, ainda não existem dados conclusivos que justifiquem a maior ocorrência de casos de PFP nos períodos mais frios do ano.

Condições associadas, tais como: hipertensão arterial, diabetes mellitus, viroses, gravidez e puerpério aumentam os riscos de PFP. Dos casos observados neste estudo, em que havia como comorbidade prévia a HAS, foi verificado em $46 \%$ destes, associações com outras doenças. Em outros estudos foi relatado maior prevalência da PFP com etiologia desconhecida. ${ }^{(8)} \mathrm{Em}$ uma investigação para determinar a etiologia e fatores de risco associados da PFP, em Lima, Peru onde houve um surto de síndrome Bell, não foi observado associação clara ou risco identificável comum nos casos analisados ${ }^{(24)}$

O início da reabilitação na fase aguda dos sintomas pode estar associado ao melhor prognóstico ou manutenção do grau de comprometimento do nervo facial. ${ }^{(12)}$ Dos casos avaliados na unidade de 
internamento, $86 \%$ destes foi encaminhado para a fisioterapia durante a permanência no hospital. Todavia, do total dos pacientes atendidos, $15 \%$ não receberam encaminhamentos para continuidade do tratamento no período pós-alta hospitalar. Nesta unidade em questão, o encaminhamento para fisioterapia tanto durante a internação como no pós -alta é realizado pelo médico. Observou-se neste estudo a necessidade de estabelecer um fluxograma para reabilitação dos casos de PF durante o internamento na unidade e também no pós-alta. Embora o tratamento precoce da fisioterapia melhore consideravelmente o prognóstico da reabilitação, a maioria dos encaminhamentos é realizada quando existe uma complicação instalada, o que diminui a possibilidade de recuperação. ${ }^{(12)}$

Diferentemente de outros trabalhos, ${ }^{(4,}{ }^{26)}$ neste estudo não foram observadas queixa em relação à aparência devido a PF (Tabela 1). Um dos fatores que poderia explicar a ausência desta queixa é a associação com outras comorbidades ou maior envolvimento motor incapacitante como a hemiplegia associada à AVE. Porém, nem sempre o resultado da avaliação é condizente com a percepção do paciente em relação ao impacto da paralisia facial.(25)

Com relação ao comprometimento motor, as PFC classificadas de acordo com a escala de HB, como paralisia grave ou paralisia total, foram predominantes no quadrante inferior da face (Tabela 2). Este fato também foi constatado em outros trabalhos, onde os músculos faciais do quadrante superior foram menos afetados. ${ }^{(2,14)} \mathrm{O}$ comprometimento motor no quadrante superior da face foi observado em nosso trabalho, porém em pequena proporção, e isto pode estar relacionado às posições variáveis das lesões cerebrais não observadas durante a coleta de dados sendo em nosso estudo classificadas em AVEi e AVEh. Entretanto, existe escassez de trabalhos científicos que atentem a explorar os conhecimentos da diminuição do tônus muscular no quadrante inferior e em menor proporção o quadrante superior da face nos casos da PFC decorrente de AVE.

\section{CONCLUSÃO}

Devido o estudo ter sido realizado em uma unidade de internamento de alta complexidade, a incidência da PFC foi maior em relação à PFP, bem como o alto índice de HAS entre as doenças prévias diagnosticadas e também a associação da PF com outras comorbidades. Não foi constatada queixa em relação à aparência devido a PF. A PF tanto de origem central como periférica apresentou característica sazonal com maior prevalência dos casos nos meses de abril a julho. Apesar de existir encaminhamento à fisioterapia foi observada a necessidade de estabelecer um fluxograma para reabilitação dos pacientes durante o internamento e no pós-alta hospitalar.

\section{REFERÊNCIAS}

1. Santos-Lasaosa S, Pascual - Millán LF, TejeroJust C, Moralis-Asín F. Parálisis facial periférica: etiologia, diagnóstico y tratamiento. Rev Neurol. 2000;11:1048-53.

2. Correa, CL. Paralisia facial periférica. São Paulo: Phorte Editora; 2011.

3. Lazarini PR, Fernandes AM, Brasileiro VS, Custódio SE. Paralisia facial periférica por comprometimento do tronco cerebral - a propósito de um caso clínico. Rev Bras Otorrinolaringol. 2002;68(1):14O-4.

4. Stuart RM, Byrne PJ. The importance of facial expression and the management of facial nerve injury. Neurosurg Q. 2004;14(4):239-48.

5. Mahadevappa K, Vora A, Graham A, Nesathurai S. Facial paralysis: a critical review of accepted explanation. Med Hypotheses. 2010;74(3):5089.

6. Calais LL, Gomez M, Bento RF, Comerlatti LR. Avaliação funcional da mímica na paralisia facial central por acidente cerebrovascular. Prófono. 2005;17(2):213-22.

7. Sanvito WL. Propedêutica neurológica básica. 2ed. Atheneu São Paulo; 2010.

8. Vasconcelos BEC, Dias E, Dantas WRM, Barros ES, Monteiro GQM. Paralisia facial periférica 
traumática. Rev Cir Traumat Buco-Maxilo-Facial. 2001;1(2):13-2O.

9. Garanhani MR, Cardoso JR, Capelli A de G, Ribeiro MC. Fisioterapia na paralisia facial periférica: estudo retrospectivo. Rev. bras. otorrinolaringol. 2007;73(1):112-5.

10. Goffi-Gomez MVS, Vasconcelos LGE, Moraes M. Trabalho miofuncional na paralisia facial. Arq Fund Otorrinolaringol. 1999;3(1):30-4.

11. Ribeiro EC, Cassol M. Enfoque fisioterápico e fonoaudiólogo na paralisia facial periférica. Arq Fund Otorrinolaringol. 1999;3:46-52.

12. Tessitore A, Paschoal JR, Pfeilsticker LN. Avaliação de um protocolo da reabilitação orofacial na paralisia facial periférica. Revista CEFAC. 2009;11(3):432-40.

13. House JW, Brackmann DE. Facial nerve grading system. J Otolaryngol Head Neck Surg. 1985 Apr;93(2):146-7.

14. Maruska SM. Avaliação de Métodos de Tratamentos Fisioterápicos na Paralisia Facial Periférica. [Curitiba]: Universidade Federal do Paraná; 2004.

15. Saúde M da. A vigilância, o controle e a prevenção das doenças crônicas não transmissíveis: DCNT no contexto do Sistema Único de Saúde brasileiro. Organização PanAmericana da Saúde Brasília; 2005.

16. Barbara M, Antonini G, Vestri A, Volpini L, Monini S. Role of Kabat physical rehabilitation in Bell's palsy: A randomized trial. Acta Otolaryngol. 2010;13O(1):167-72.

17. Flores FP, Zazueta MR, Garcia HL. Tratamiento de la paralisis facial periférica idiopática: terapia física versus prednisona. Rev Med IMSS. 1998;36:217-21.
18. Manikandan N. Effect of facial neuromuscular re-education on facial symmetry in patients with Bell's palsy: a randomized controlled trial. Clinical Rehabilitation. 2007;21(4):338-43.

19. Almeida MAM de. Peripheral facial palsy in the city of Petrópolis. Arq. Neuro-Psiquiatr. 1992;50(1):60-4.

2O. De Diego JI, Prim MP, Madero R, Gavilan J. Seasonal patterns of idiopathic facial paralysis: a 16-year study. J Otolaryngol Head Neck Surg. 1999;12O(2):269-71.

21. Jakovljevi D, Salomaa V, Sivenius J, Tamminen M, Sarti C, Salmi K, et al. Seasonal Variation in the Occurrence of Stroke in a Finnish Adult Population The FINMONICA Stroke Register. J Stroke. 1996 Oct 1;27(10):1774-9.

22. Wang $X Y$, Barnett $A G$, Hu W, Tong $S$. Temperature variation and emergency hospital admissions for stroke in Brisbane, Australia, 1996-2005. Int J Biometeorol. 2009;53(6):53541.

23. Brennan PJ, Greenberg G, Miall WE, Thompson SG. Seasonal variation in arterial blood pressure. Br Med J (Clin Res Ed). 1982;285(6346):919.

24. Reaves, EJ; Ramos, M.; Bausch DG. Workplace cluster of Bell's palsy in Lima, Peru. BMC Res Notes. 2014,7:289.

25. Freitas KCS, Goffi-Gomez MV. Grau de percepção e incômodo quanto à condição facial em indivíduos com paralisia facial periférica na fase de sequelas. Rev Soc Bras Fonoaudiol. 2008;13(2):113-8.

26. Santos RMM; Guedes ZCF. Estudo da qualidade de vida em indivíduos com paralisia facial periférica crônica adquirida. Rev. CEFAC. 2012 Jul-Ago; 14(4):626-634. 